\title{
Supraspinal Nocifensive Responses of Cats: Spinal Cord Pathways, Monoamines, and Modulation
}

\author{
KENNETH L. CASEY AND THOMAS J. MORROW \\ Deparments of Neurology (K.L.C.) and Physiology (K.L.C., T.J.M.), \\ University of Michigan, Neurology Research Laboratories (Neurophysiology), \\ Veterans Administration Medical Center, Ann Arbor, Michigan 48105
}

\begin{abstract}
These experiments were conducted to determine (1) whether dorsal and ventral ascending spinal pathways can each mediate unlearned supraspinal nocifensive responses of cats to noxious thermal stimuli and (2) whether interrupting the spinal projection of supraspinal monoaminergic neurons alters the excitability and natural modulation of these responses. In partially restrained cats, thermal pulses $\left(\geqslant 47^{\circ} \mathrm{C}\right)$ delivered to the hindlimbs of intact cats or rostral to lesions of the thoracic spinal cord elicited abrupt body movements and interruption of eating (or of exploring for) liquified food. These electronically monitored responses automatically terminated the stimulus. Natural modulation of responsiveness was produced by delivering food and thermal stimuli simultaneously; this reduced response probability by an average of $41 \%$. Complete transection of the thoracic spinal cord eliminated both thermally elicited responses and orienting responses to noxious and tactile mechanical stimulation of the hindlimbs.

Ventral bilateral thoracic spinal cord lesions that spared only the dorsal funiculus and portions of the dorsolateral funiculus (three cats) significantly reduced orienting responses to all mechanical hindlimb stimuli and reduced, but did not eliminate, movement and interrupt responses to noxious thermal hindlimb stimuli. Response latency was unaffected. Food-induced response supression persisted although lumbar spinal cord concentrations of serotonin (5HT) and norepinephrine (NE) were markedly reduced. A bilateral lesion of the dorsal funiculi and dorsal portions of the dorsolateral funiculi (one cat) also reduced nocifensive responsiveness, but only the $\mathrm{NE}$ concentration in lumbar spinal cord was reduced significantly relative to a matched cervical sample. In contrast, deep bilateral lesions of the dorsolateral funiculi (two cats) produced an increase in the probability of movement and interrupt responses without affecting either response latency or food-induced response supression. Lumbar spinal cord concentrations of $\mathrm{NE}$ and, in one cat, $5 \mathrm{HT}$ were reduced.

We conclude that (1) the dorsal and ventral spinal funiculi are each sufficient to initiate and necessary to maintain normal supraspinally organized nocifensive behavior in the cat; (2) descending monoaminergic pathways are not necessary for the phasic modulation of these responses; and (3) the tonic excitability, but not the phasic modulation, of these responses is determined in part by fibers in the dorsolateral funiculus.
\end{abstract}

Key words: pain, behavior, neurotransmitters, temperature, lesions

Our understanding of the functional significance of spinal pathways that transmit or modulate nociceptive information in the cat is based almost entirely on a study by Kennard ('54). Other reports of the sensory effect of feline
Accepted September 2, 1987. 
spinal tractotomies on pain-related behavior have been limited to brief clinical descriptions or to studies of one type of lesion (Melzack et al., '58; Levitt and Levitt, '68; Frommer et al., '77; Hayes et al., '84). Kennard's ('54) report remains the only systematic study of the effects of various spinal tractotomies on behavioral responses that may indicate the perception of pain in the cat. Unfortunately, the noxious stimulus used in that study (pinprick) was not measured and the method of application was not described; nor was there any description or measurement of the behavioral response. There is therefore a need to reexamine the question of pain-related spinal pathways in the cat, especially in view of the large number of physiological and anatomical studies of nociceptive mechanisms that have been undertaken in the past 33 years (for review, see Dennis and Melzack, '77; Kerr and Casey, '78; Willis and Coggeshall, '78; Yaksh and Hammond, '82; Dubner and Bennett, ' 83 ; Vierck et al., '83; Willis, '85).

Most behavioral studies of pain-related responses in animals have used spinal segmental reflexes, which can be elicited without intact supraspinal or suprabulbar mechanisms (for review see Chapman etl al., '85). To understand more fully the functional significance of spinal sensory pathways, and in particular their possible role in pain, it is necessary to use well-controlled noxious stimuli and to monitor nonreflexive responses that may reasonably be assumed to reflect the supraspinal organization of nocifensive behaviors. We have developed a method for determining the probability that a controlled thermal stimulus of the cat hindlimb will elicit, within a determined latency, one or more unlearned nonreflexive responses (Casey and Morrow, '83). This method has been used in the present study to determine how various thoracic spinal tractotomies affect nonreflexive, supraspinal nocifensive behaviors in the cat.

We wished to determine whether pathways ascending in the dorsal or ventral spinal cord of cats were necessary for maintaining normal supraspinal nocifensive responses and whether these same pathways were sufficient to initiate them. We also wished to determine whether the interruption of supraspinal descending pathways, which have been shown to modulate the nociceptive responses of dorsal horn neurons (Soja and Sinclair, '83) by monoaminergic mechanisms (for review, see: Mayer and Price, '76; Messing and Lytle, '77; Basbaum and Fields, '78; Willis and Coggeshall, '78; Dubner and Bennett, '83), would affect baseline nocifensive responsiveness or the natural modulation of responsiveness (Pavlov, '27; Casey and Morrow, '83) in conjunction with changes in spinal cord concentrations of serotonin (5HT) and norepinephrine (NE).

We have used the minimum number of animals necessary to test our hypotheses because of the severe motor disability produced by most of the tractotomies. Preliminary reports of some of these results appear elsewhere (Casey et al., '81, '83, '87).

\section{MATERIALS AND METHODS}

All animals in this study were maintained in facilities that were fully accredited by the American Association for Accreditation of Laboratory Animal Care.

\section{Testing of responses to thermal stimuli}

Seven normal adult female cats were trained to place their muzzles into an illuminated food cup, thereby interrupting a photocell contact and triggering the delivery of three $1.5-\mathrm{ml}$ portions of pureed meat-based cat food during a 15-second period. After 1-2 weeks of preliminary conditioning, the cats were trained for another 2 weeks to perform the same task while partially restrained in a flexible body cast. Cats were food deprived for 24 hours before training or data-collection sessions 5 days per week.

Thermal pulses were delivered to either shaved outer thigh by spring-loaded, water-cooled contact thermodes (Morrow and Casey, '81). Glycerine was applied to the skinthermode interface to assist heat transfer, and an electric razor was used to shave the skin twice weekly. Stimuli rose from an adapting temperature of $38^{\circ} \mathrm{C}$ at a rate of $17^{\circ} \mathrm{C} \cdot \mathrm{s}^{-1}$. Unless terminated by the cat's response, thermal pulses had a plateau duration of 5 seconds and returned to the adapting temperature within 2 seconds.

Three behavioral responses were recorded only during the 7-seconds stimulus period. Vocalization was detected by microphone, movement by a weighted phonograph cartridge mounted between the hindlimb supports, and interruption of eating by activation of a photocell mounted in the illuminated foodcup. The amplified output of each of these devices was led into separate level-detection comparator circuits, each with an output that could be selected to terminate the stimulus. Movement responses sufficient to trigger the detector produced just-visible displacements of the cast and registered $300 \mathrm{~g}$ peaks on a force transducer. The movement sensor could be activated by hindlimb or upper body movements but not by the head movements that signalled interruption of eating.

A programmable integrated circuit controlled the feeding schedule, timing, laterality, and intensity of thermal stimuli and the activation of three response-latency counters. Periodic illumination of the food cup signaled the onset of one of 43 fifteen seconds food-available periods, 32 of which were used for data collection. The program advanced only when the cat's muzzle entered the food cup. Interruption of photocell contact triggered stimuli on 22 of the food-available periods so that thermal stimulation was coincident with eating; ten stimuli were noncoincident. Interrupt responses to noncoincident stimuli occurred when the cat's muzzle was in the empty food cup. During each session, 12 stimuli were blank trials at $38^{\circ} \mathrm{C}, 12$ were at an intermediate temperature, and eight were at the highest temperature selected for that session. Four of the blank and intermediate stimuli and two of the higher-intensity stimuli were not coincident with eating. The length of the interstimulus interval depended on the rate at which each cat advanced the program by responding to the illuminated food cup. The shortest possible session was approximately 30 minutes long, giving a minimum interstimulus interval of 1.5 minutes (not including blank trials). Some cats took 1 hour to advance through the program, thus doubling the average interstimulus interval. The stimulus sequence was based on a random order. (Fellows, '67) of intensity and laterality, modified to assure that high-intensity pulses could not be delivered to the same site at intervals shorter than 3 minutes. The frequency, duration, and intensity of noxious stimuli delivered according to the above schedule were well within tolerance because all cats readily accepted daily placement in the apparatus and regularly activated advancement of the stimulus delivery program.

At stimulus onset, three response-latency counters were started. A pulse from a comparator circuit stopped the appropriate counter when the output of the microphone or movement detector amplifier exceeded a selected level. Photocell activation stopped the interrupt-detection counter 
when the cat's muzzle was withdrawn from the food cup. Response latencies were read from the digital display. Any response could abort the stimulus.

\section{Data analysis}

Response latencies between 0.5 and 7 seconds after each stimulus pulse onset were entered onto disk files of a Data General Nova 3/12 laboratory computer for the computation of response probabilities, the mean $\pm \mathrm{SD}$ of the response latency for each stimulus temperature, and the construction of response latency histograms for each temperature trial. Stimulus laterality was identified. The variance about each response probability was expressed as the standard error, and significant differences between probabilities were determined by computing the $95 \%$ confidence intervals of each probability (Freund, '62). For probabilities based on less than 30 trials, we used the tables computed by Crow ('56). Responses to stimulation of each side were analyzed separately and compared. Responses to coincident and noncoincident stimuli were also analyzed separately; a significant difference between these probabilities was interpreted as food-induced response modulation. Analyses of response trends were obtained by sequentially computing the response probabilities for each temperature in blocks of 3 days.

\section{Surgery and postoperative care}

Each cat was deeply anesthetized with $50 \mathrm{mg} / \mathrm{kg}$ of pentobarbital I.V. Supplemental doses were given as needed to maintain complete suppression of flexor reflexes to intense mechanical stimuli.

Under aseptic conditions, a complete vertebral laminectomy was performed over spinal cord segment $T_{6-8}$. Dural flaps were created bilaterally to rotate the exposed spinal cord for inspection or surgery as needed during the lesion procedure. For ventral cord lesions, care was taken to avoid the anterior spinal artery; in two cats, fine sutures were used to ligate dorsal spinal arteries and veins before creat ing a dorsal cord lesion. Immediately before and after creating a lesion, the exposed spinal cord was flooded with sterile iced saline. Lesions were made with iridectomy scissors, pieces of razor blade, and the beveled edges of 25gauge hypodermic needles. In one cat $(\mathrm{P})$, the entire spinal cord except for the dorsal columns was completely severed by garroting the tissue with nylon suture.

Each cat was given antibiotics intramuscularly daily for 1 week after surgery and received sterile water subcutaneously during the immediate postoperative recovery period, as needed. Special longer-term care was given to those cats rendered paraplegic by the lesion. Such animals required daily or twice-daily manual abdominal expression of urine and feces, and careful attention was paid to the grooming and conditions of the hindlimbs. Except for the development of hindlimb atrophy, cats maintained their body weight and normal food consumption, appeared active and alert, and responded affectionately to petting and handling. All cats exercised freely outside their cages daily.

At least 3 weeks of recovery were allowed before behavioral testing. Three cats received spinal cord lesions while naive; two cats ( $\mathrm{M}$ and $\mathrm{W}$ ) were tested with thermal stimuli caudal to the lesion before being tested with rostral stimuli delivered at approximately the $\mathrm{T}_{3-5}$ dermatomes according to Hekmatpanah ('61). The third cat had a complete transection of the thoracic spinal cord and was tested for orien- tation responses to mechanical stimuli (see below) and for responses to thermal stimulation of the hindlimbs.

\section{Testing of tactile and intense mechanical stimuli}

After their spinal cord surgery, five cats were tested for orientation responses to mechanical stimuli delivered manually to each upper forelimb or hind paw. Each cat was placed on a table facing away from the experimenter, who was positioned directly behind the cat. While the cat was looking straight ahead, one of four types of stimuli were delivered outside the cat's visual field: light touch, firm skin pinch, firm muscle squeeze, or repetitive pinprick. The latter three stimulus types were applied with an intensity that was unequivocally noxious when applied to the experimenter's hand or arm. To partially control for the tactile cues of noxious mechanical stimuli, both hindlimbs or hindpaws were held gently before applying the intense stimulus to one side. Each stimulus was applied for 3 seconds or until the cat turned its head to the side of the stimulus. The numbers of each type of stimulus and of each response were separately recorded for right and left sides. Vocalizations that occurred without orientation during intense stimuli were also counted as responses, but head movements to the side opposite the stimulus were recorded and counted as nonresponses. A $2 \times 2$ contingency chi-square test was used to detect significant differences in the proportion of correct orientation responses to forelimb, as compared to hindlimb, stimuli of each type.

\section{Histology}

The cats were deeply anesthetized with pentobarbital and perfused through the heart with normal saline and $10 \%$ formalin in saline. The lesioned portion of the thoracic spinal cord was exposed for approximately $20 \mathrm{~mm}$ rostral and caudal to the lesion site. The exposed portion was carefully removed and placed in $10 \%$ formal saline for 1 week before sectioning on a freezing microtome. After removing the dura, 50 frozen sections were cut sagittally through a $20-\mathrm{mm}$ block of tissue with the lesion in the middle. The remaining $10-\mathrm{mm}$ segments rostral and caudal to the lesion were coronally sectioned at $50-\mu \mathrm{m}$. In one cat (P), only coronal sections were cut because of extensive cavitation of the spinal cord ventral to the dorsal columns, which appeared intact on inspection under a dissecting microscope. Both sagittal and coronal sections were stained alternately by the Nissl and Weil-Weigert methods. The location and extent of the lesion in each case were determined by inspection of the projected images of both the sagittal and coronal sections. The degeneration of pathways interrupted by the lesion was confirmed by the presence of gliosis seen on the Nissl-stained coronal sections and the relative lack of myelin staining on the Weil-Weigert-stained sections.

\section{Biochemical assays}

Before transcardiac perfusion, $10-\mathrm{mm}$ sections of the cervical and lumbar spinal cord were removed for biochemical analysis. Similar control samples were taken from seven unoperated cats. Tissue levels of $5 \mathrm{HT}$ and NE were determined by high-performance liquid chromatography and electrochemical detection according to the technique described by Terry and Craig ('85). Levels of 5HT and NE are expressed as ng/g tissue. 


\section{RESULTS \\ Overview}

The results are summarized in Figure 1. Details are given in the text that follows. The cat with a complete spinal cord transection showed no responses to mechanical or thermal stimulation of the hindlimbs. Ventral thoracic spinal cord lesions that spared the dorsal column and some dorsolateral funicular fibers (cats $\mathrm{C}, \mathrm{W}$, and $\mathrm{P}$ ) reduced, but did not eliminate, supraspinal nonreflexive nocifensive responses. Response latency was unaffected. Such lesions resulted in a marked drop in 5HT and NE levels in the lumbar spinal cord but did not interfere with the food-induced suppression (modulation) of responses. A complete lesions of the dorsal columns and dorsal dorsolateral fibers (cat M) also reduced, but did not eliminate, nocifensive responses and had no effect on response latency, response suppression, or 5HT levels in the lumbar spinal cord; NE levels were slightly reduced. However, two dorsal lesions that extended ventrally to involve most of the dorsolateral quadrants (cats $\mathrm{S}$ and $\mathrm{L}$ ) resulted in increased nocifensive responsiveness. Response latency was unaffected. Lumbar $5 \mathrm{HT}$ levels were reduced in one cat, $\mathrm{NE}$ levels were slightly reduced, and food-induced response suppression was not affected in these cats.

\section{Preoperative and above lesion testing}

An average of $74 \pm 2$ (SD) coincident (with food) and 31 \pm 8 (SD) noncoincident stimuli were delivered to each cat at each temperature. The control observations were consistent with previous results (Casey and Morrow, '83). All cats showed a significant increase in the probability of one or more nocifensive responses as stimulus temperature in creased. None of the cats emitted a significant number of vocalization responses. Responsiveness did not differ with stimulus laterality with the exception of one behavior in one cat; this is discussed below.

We define threshold as the lowest stimulus temperature at which the response probability exceeds that at $38^{\circ} \mathrm{C}$ by the larger of either the lower $95 \%$ confidence limit at the test temperature or the upper $95 \%$ confidence limit at $38^{\circ} \mathrm{C}$. This confidence limit criterion is also used to define significant differences between response probabilities at other temperatures. By this definition, all cats had nocifensive response thresholds of $47^{\circ} \mathrm{C}$ for one or more behaviors.

As in our previous study (Casey and Morrow, '83), response latency was defined as the time from the beginning of the thermal pulse plateau to the onset of the behavioral response. In the present study, we found the average response latency of all cats to range from $3.2 \pm 0.2$ (SE)

SUMMARY: Effect of Lesions of Thoracic Spinal Cord

\begin{tabular}{|c|c|c|c|c|c|c|}
\hline Cat & Lesion & Non-refle & Nocife & ve Response & Lumbe & 5HT, NE \\
\hline & & Present & Change & Modulated & $5 \mathrm{HT}$ & $\mathrm{NE}$ \\
\hline C & & YES & & YES & & \\
\hline$n$ & & YES & & YES & & \\
\hline $\mathbf{P}$ & & YES & & $\operatorname{YES}(\downarrow)$ & & \\
\hline M & & YES & & YES & NS & 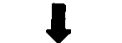 \\
\hline L & & YES & & YES & NS & 1 \\
\hline $\mathbf{s}$ & & YES & & YES & 1 & 1 \\
\hline
\end{tabular}

Fig. 1. Summary of results. Location and extent of thoracic spinal cord lesions are suggested by crosshatching on symbols in left column. Histology of lesions is shown in Figure $4 \mathrm{~A}$ and $\mathrm{B}$. Cat with complete transection (no shown) showed no nocifensive responses to noxious hindlimb stimuli. Large arrows indicate direction of statistically significant changes in one or more responses to noxious thermal stimuli or in the lumbar spinal cord concentrations of $5 \mathrm{HT}$ and $\mathrm{NE}$ relative to the average of seven control samples of lumbar spinal cord. Small arrows indicate a decreased modulation (cat P) or a decreased lumbar NE or $5 \mathrm{HT}$ concentration relative to a paired sample from the cervical spinal cord. NS = no statistically significant change. 


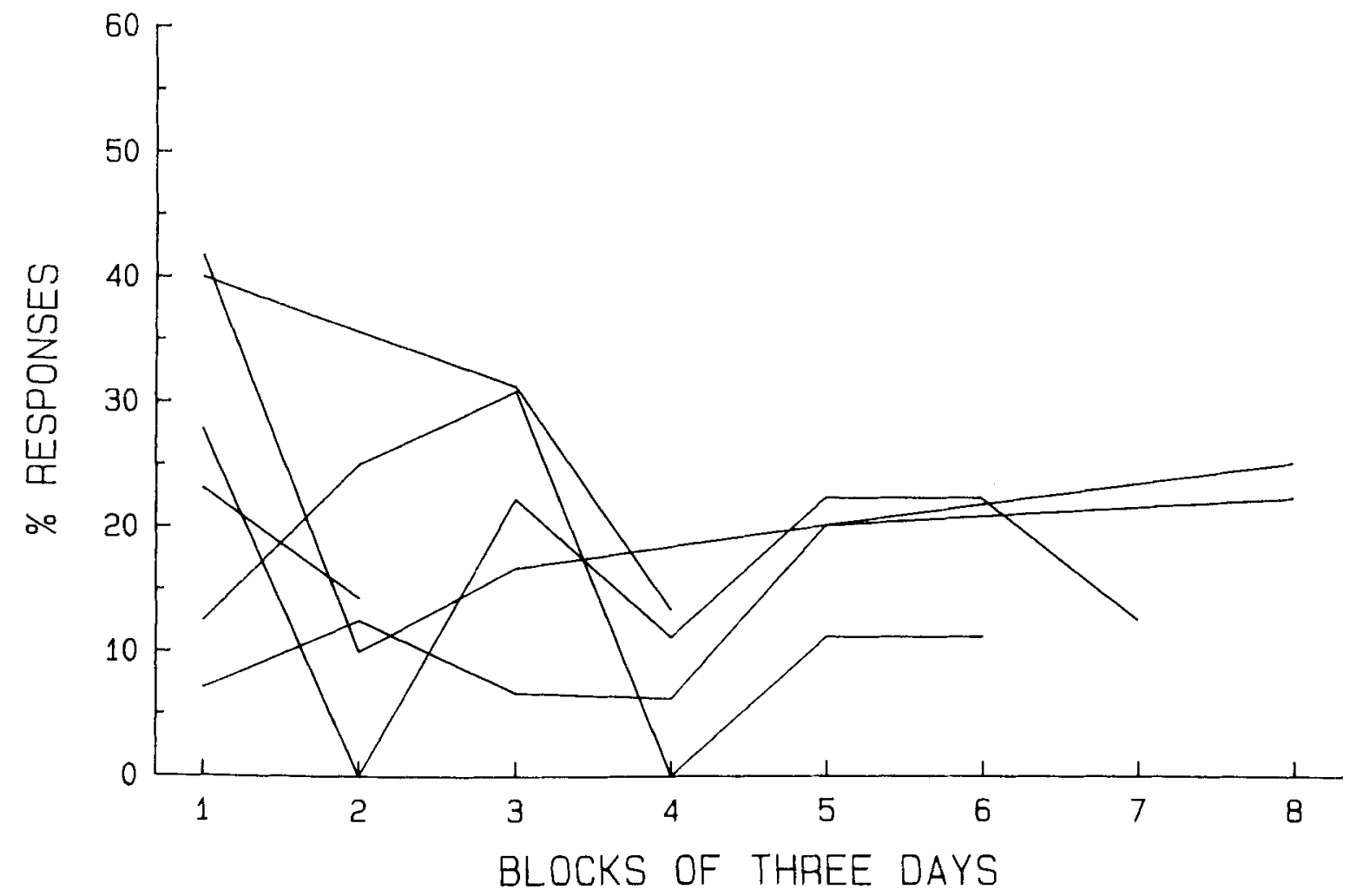

Fig. 2. Sequential responsiveness to coincident noxious thermal stimuli that were threshold for the behavior (interrupt or movement) and were delivered for at least six sessions in sequence. Temporary technical problems interrupted sequential testing of two cats.

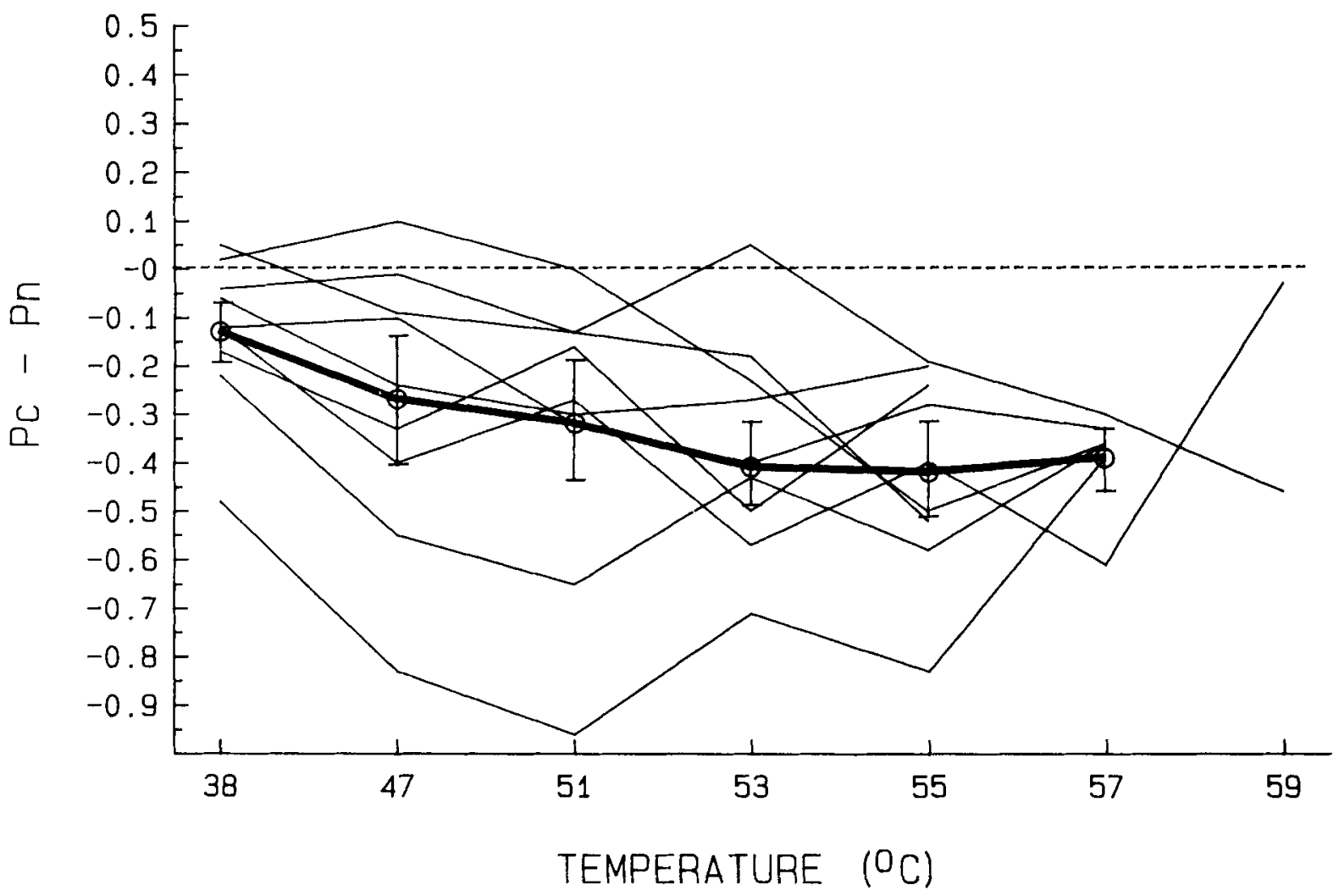

Fig. 3. Food-induced supression of nocifensive behaviors of all six cats. The ordinate indicates the difference between the probability of a response to a thermal stimulus delivered coincident with food $(\mathrm{Pc})$ and the probability of the same response to a noncoincident stimulus $(\mathrm{Pn})$. Nine modulation profiles are shown because three cats modulated only one behavior. Heavy line with open circles shows average difference $\pm \mathrm{SE}$. 
seconds for 304 interrupt responses to coincident stimuli to $2.3 \pm 0.2$ (SE) seconds for 206 movement responses to noncoincident stimuli. An analysis of variance reveals a significant difference among the latencies of the four types of responses $(\mathrm{F}[\mathrm{df}=3]=3.31 ; P<.05)$, but not among cats. This difference among response latencies is attributable to the longer interrupt response latencies to coincident stimuli obtained in this study as compared with those obtained previously from a larger sample (Casey and Morrow, '83). In accord with previous results, we did not detect a significant relationship between response latency and stimulus intensity.

We determined the response probability for sequential blocks of 3 days for each stimulus intensity delivered to each cat. Both individual and combined plots of the different responses of each cat to the threshold temperature (Fig. 2) show no apparent trend of responsiveness during preoperative testing or during testing above the level of the lesion. Correlation coefficients for the pooled data of Fig, 2 $(-0.2714, \mathrm{~d} \mathbf{F}=5,14)$ and for each of three cats with at least six successive 3-day blocks of tests at threshold $(0.5235$, $(-0.0122,-0.3843 ; \mathrm{dF}=5)$ are not significant $(P>.10)$.

Each cat modulated at least one nocifensive response as shown by a significantly lower response probability when the delivery of food was coincident with thermal stimulation. Three cats showed food-induced suppression of both interrupt and movement responses; of the three remaining cats, two suppressed interrupt responses and the other showed suppressed movement responses only. Fig. 3 shows the average and individual modulation profiles of all foodmodulated behaviors of all cats expressed as the difference between response probabilities to noncoincident and coincident stimuli. This naturally induced modulation suppresses responsiveness to stimuli in the $53-57^{\circ} \mathrm{C}$ range by an average of $41 \pm 1.2 \mathrm{SD} \%$ (Fig. 3 ).

\section{Postoperative and below-lesion testing}

The cat with a complete transection of the thoracic spinal cord showed no responses to 131 mechanical or 74 noxious thermal $\left(55^{\circ} \mathrm{C}\right)$ hindlimb stimuli.

Response latency did not change significantly for any behavior of any cat. An analysis of variance showed no significant difference between average preoperative and postoperative latencies $(\mathrm{F}(\mathrm{d}=1)=0.036 ; P=.851)$. Indeed, the range of average postoperative latencies was nearly identical to the average preoperative range: $3.3 \pm 0.2$ (SE) for interrupt responses to coincident stimuli to $2.4 \pm 0.2$ (SE) for interrupt responses to noncoincident stimuli.

Postoperative changes in responsiveness were associated with lateralized differences in responsiveness in only one behavior of one cat, as discussed below.

Ventral lesions. Three cats received ventral lesions of the thoracic $\left(\mathrm{T}_{6-8}\right)$ spinal cord (Fig. 4). Clinical evaluation of each cat revealed a complete and persistent loss of bowel and bladder control as evidenced by the need to manually express bowel and bladder contents daily. Each cat had complete spastic paralysis of the hindlimbs.

Tests of mechanical sensory function (see Materials and Methods) showed that cat $C$ had a significant $\left(\chi^{2}=9.75 ; P\right.$ $<.01$ ) loss of orientation responses only to pinprick caudal to the lesion (45\% of 22 stimuli) as compared to rostral to the lesion (100\%) of 15 stimuli). In separate analyses, cat $\mathrm{W}$ showed a significant $\left(\chi^{2}=14.5-28.8 ; P<<.01\right)$ loss of responsiveness to skin pinch, muscle pinch, and pinprick delivered caudal to the lesion; tactile responsiveness may have been preserved, but only 11 caudal stimuli were tested. Cat $P$ was not tested for responses to mechanical stimuli.

Tests of responses to hindlimb thermal stimuli showed that all three cats had significantly reduced but persistent interrupt responses and two cats (C and $P$ ) also showed interrupted responses and two cats $(C$ and $P$ ) also showed reduced but persistent movement responses. Figure 5 shows the differences produced by these lesions in the profiles of the interrupt and movement responses to coincident thermal stimuli. These findings are based on an average of 70 \pm 22 (SD) coincident and $32 \pm 10(\mathrm{SD})$ noncoincident preoperative or above-lesion stimuli and an average of $87 \pm 11$ (SD) coincident and $38 \pm 7$ (SD) noncoincident postoperative or below-lesion stimuli delivered at each temperature to each of these three cats.

Cat $\mathrm{C}$ showed a lateralized difference postoperatively in movement responses only to coincident stimuli ( $8 \%$ of 132 left stimuli and $20 \%$ of 136 right stimuli $\geqslant 47^{\circ} \mathrm{C}$ ); however, the same difference was present in this cat during preoperative testing (18\% of 73 left stimuli and $32 \%$ of 79 right stimuli $\geqslant 47^{\circ} \mathrm{C}$ ). Furthermore, the bilateral lesion produced a similar drop in responsiveness on each side (10 and 12\%).

Response thresholds increased by as much as $2-6^{\circ} \mathrm{C}$ for one or more behaviors for each cat. However, cats $\mathrm{C}$ and $\mathrm{W}$ each showed no change in response threshold for at least one behavior for which responsiveness was decreased at higher stimulus temperatures.

Postoperative or below-lesion response probabilities were plotted in blocks of 3 consecutive days and compared with the same analysis of preoperative or above-lesion data. In the two cats ( $\mathrm{C}$ and $\mathrm{P}$ ) tested sequentially preoperatively and postoperatively on the hindlimbs only, the decreased postoperative responsiveness could not be attributed to a preoperative trend. To further examine the effect of testing duration and sequence, cat $\mathrm{W}$ was tested only after surgery, and the responses to hindlimb stimulation were tested before testing stimuli rostral to the lesion. The results (Fig. 6) show that the hindlimb responses were reduced when the usual testing sequence was reversed.

The cats with the most extensive dorsolateral funiculus sparing ( $\mathbf{C}$ and $\mathrm{W}$ ) continued to show food-induced modulation of interrupt responses with the same degree of suppression as in control trials (Fig. 7). Cat P strongly modulated movement responses to all four intensities of preoperative hindlimb stimulation. After a lesion that spared only the dorsal column and possibly some fibers in Lissauer's tract (Fig. 4), food-induced suppression was eliminated at most test temperatures but persisted at $51^{\circ} \mathrm{C}$ (Fig. 7).

Dorsal lesions. Three cats received lesions that were restricted to the dorsal half of the thoracic spinal cord $\left(\mathrm{T}_{6-}\right.$ 8) (Fig. 4). Cat $M$ underwent surgery before behavioral testing. On clinical evaluation, this cat appeared normal except for possible slight hindlimb ataxia on narrow surfaces. Cat $L$ had a stiff hindlimb gait with increased tone and intermittent ankle clonus. Cats $M$ and $L$ had intact bowel and bladder function. Cat $\mathrm{S}$ had spastic paraparesis and temporary loss of bowel and bladder control; some degree of voluntary hindlimb movement apparently returned by approximately 3 weeks postoperatively.

The orienting responses of cats $S$ and $M$ to skin pinch, muscle squeeze, and tactile stimuli caudal to the lesion were not significantly affected. However, both cats gave significantly $\left(16<\chi^{2}<19 ; P<<.01\right)$ fewer responses to hindlimb pinprick (cat M, 26\% of 19 stimuli; cat S, $49 \%$ of 57 stimuli) than to forelimb pinprick (cat M, $95 \%$ of 22 stimuli; cat $\mathrm{S}, 92 \%$ of 38 stimuli). 

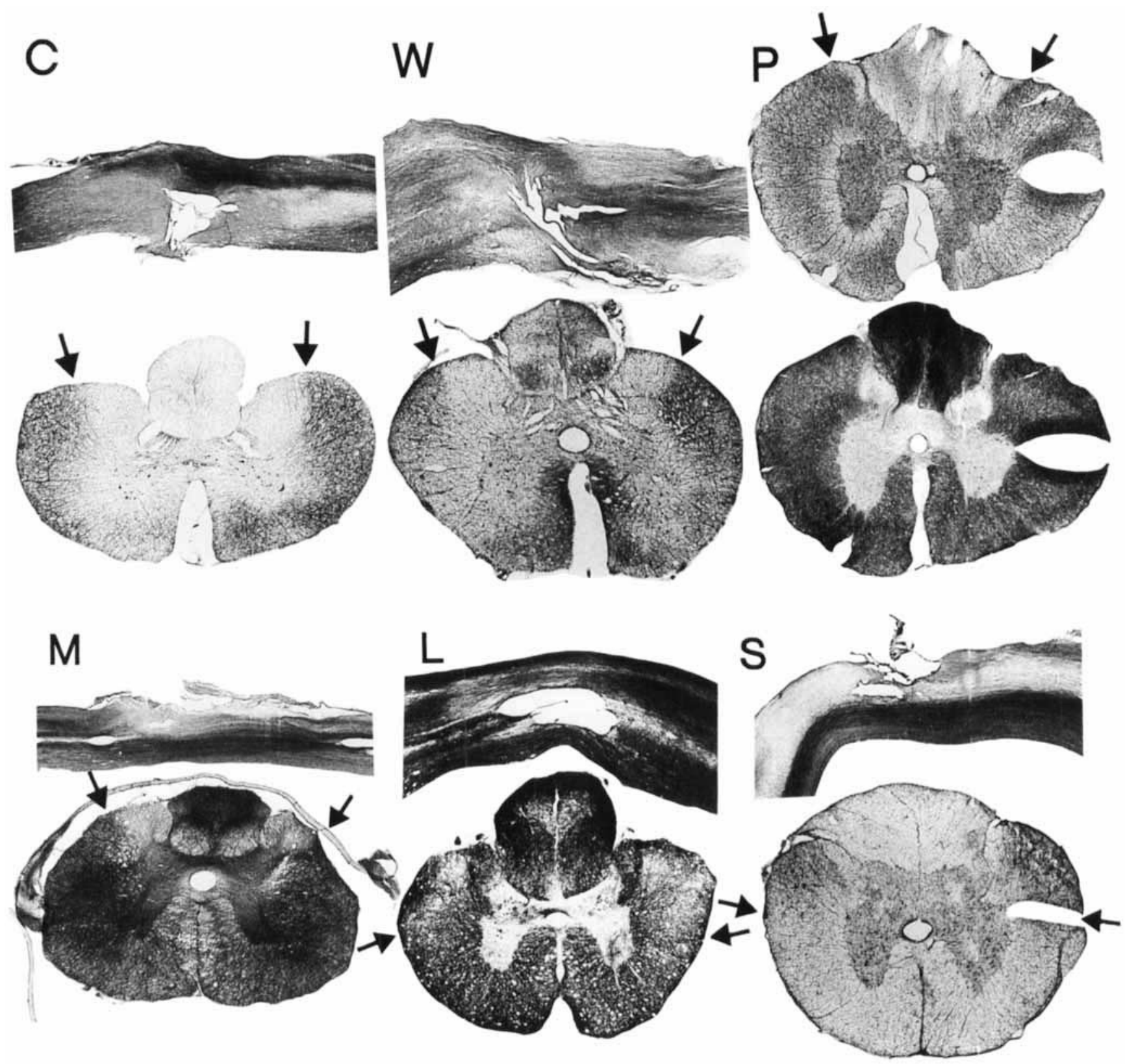

Fig. 4. Location and extent of partial transections of thoracic spinal cord. Animal identification shown at upper left of sections from cats with ventral lesions (top) and dorsal lesions (bottom). Each panel except P shows, above, a Weil-stained parasagittal section through the lesion and, below, a coronal section taken within 1.0 to $1.5 \mathrm{~cm}$ of the lesion. Arrows mark edge of lesion on coronal sections. Extensive ventral lesions with complete (C) and partial (W) sparing of the dorsal funiculi are shown in sagittal Weil sections and in the gliosis of rostral coronal Nissl-stained sections ( $C$ and $W$ ). Panel $P$ shows rostral coronal section only (see Materials and Methods) showing extensive

Thermal stimulation of the hindlimb revealed that cat $M$, with the least extensive dorsal lesion, gave significantly fewer interrupt and movement responses to hindlimb as compared to rostral stimuli. In contrast, cats $\mathrm{S}$ and $\mathrm{L}$, with lesions extending ventrally within the dorsolateral funicuIus, showed an increased probability of movement (cat S) and interrupt (cat $L$ ) responses. The interrupt responses of cat $S$ also showed a significant postoperative increase to $57^{\circ} \mathrm{C}$ stimuli. Cat $\mathrm{L}$ showed a reduced movement response

gliosis involving entire spinal cord ventral to the dorsal funiculi and tracts of Lissauer (Nissl stain above) and preservation of myelin staining within the dorsal funiculi (Weil stain below). Dorsal funiculi were completely severed in cats $\mathbf{M}$ and $\mathbf{S}$ (sagittal sections); extension of lesion into dorsolateral funiculi is shown by reduced myelin staining in Weil (M) and gliosis in Nissl (S) coronal sections caudal to the lesion. In cat $\mathrm{L}$, bilateral dorsolateral lesions spared most dorsal funicular fibers but caused reduced myelin staining in the dorsolateral funiculi as shown in the Weil-stained coronal section rostral to the lesion.

to $51^{\circ} \mathrm{C}$ stimuli. Figure 8 summarizes, for coincident stimuli, the major differences in the response profiles of the cats with dorsal lesions. These findings, and those reported below, are based on an average of $80 \pm 28$ (SD) coincident and $32 \pm 15(\mathrm{SD})$ noncoincident preoperative or above-lesion stimuli and an average of $90 \pm 11$ (SD) coincident and 35 \pm 12 (SD) noncoincident postoperative or below-lesion stimuli delivered at each temperature to each of these three cats. 

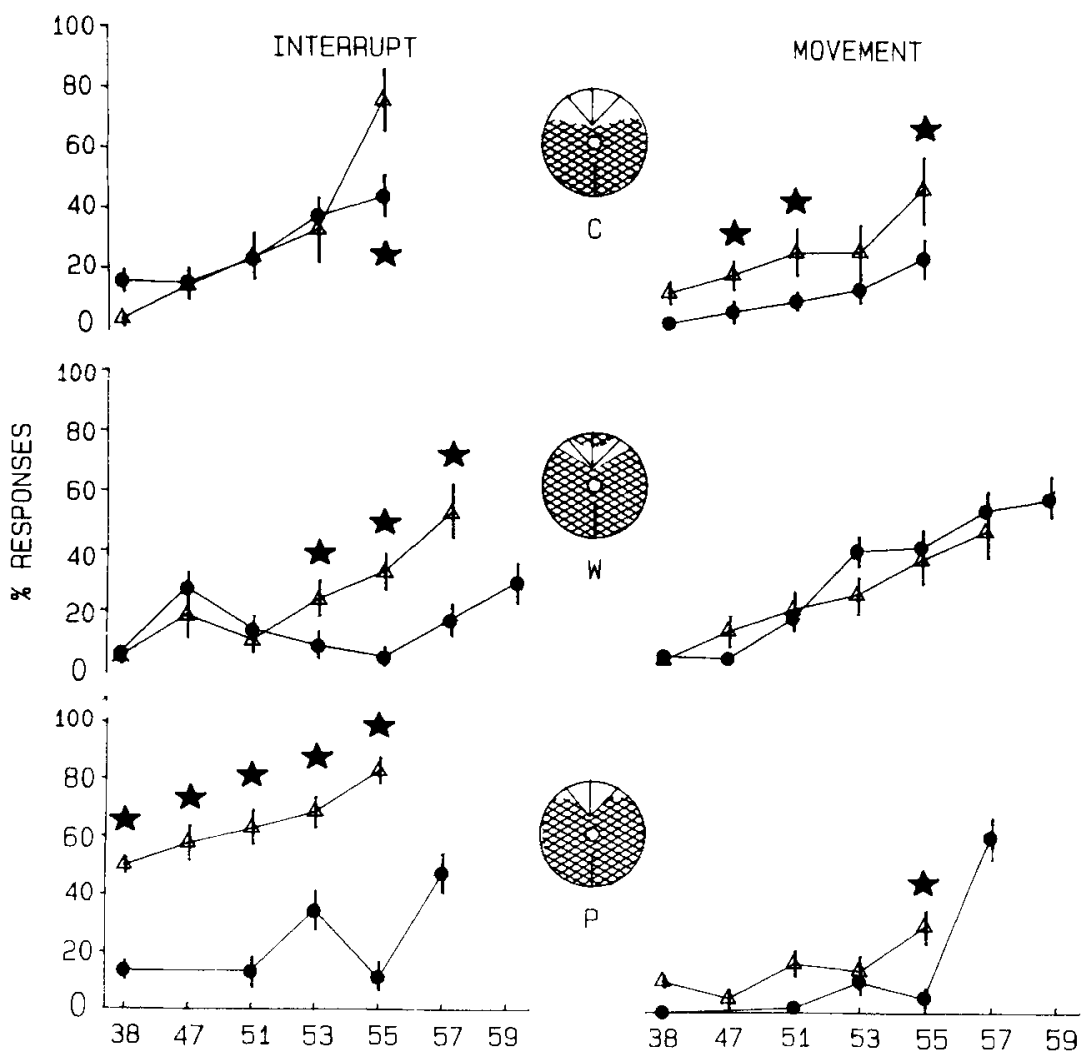

TEMPERATURE $(O \mathrm{C})$

Fig. 5. Profiles of interrupt (left) and movement (right) responses to coincident thermal stimuli. Responses to stimuli delivered preoperatively to hindlimbs (cats $\mathrm{C}$ and $\mathrm{P}$ ) or rostral to the lesion (cat W) are indicated by open triangles. Filled circles indicate responses to hindlimb stimuli deliv ered caudal to the lesion represented by crosshatching in each diagram.

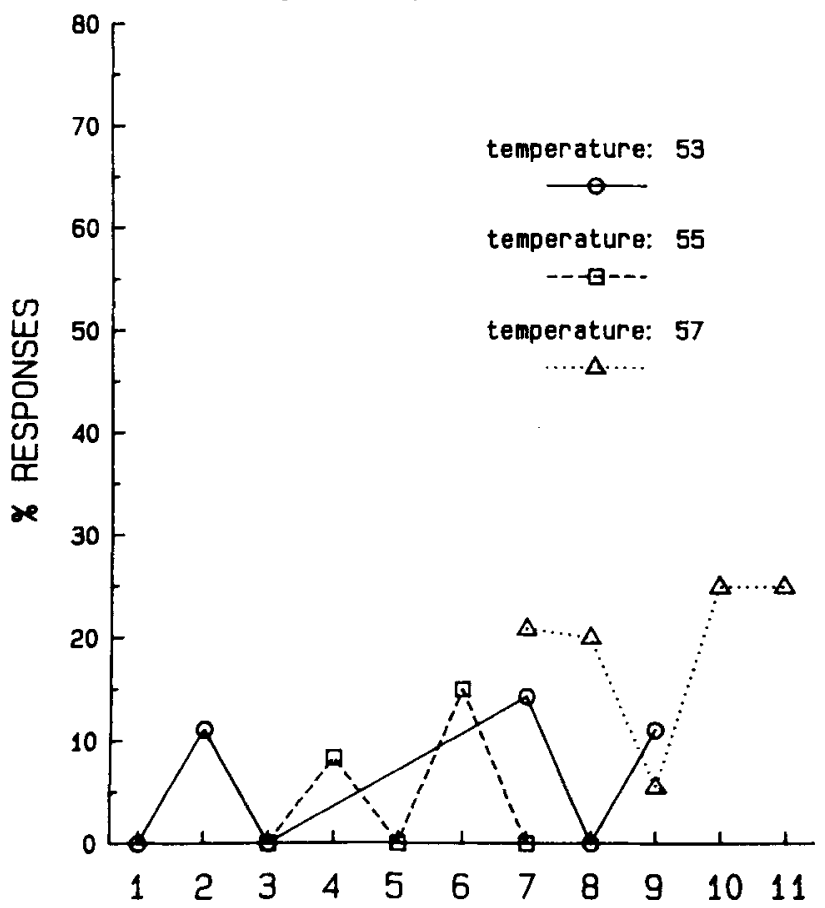

Error bars: \pm SE. Stars indicate that the \% response values at that stimulus temperature lie outside each other's $95 \%$ confidence limits. In this figure, all profiles show reduced but preserved responses to noxious thermal stimuli delivered caudal to the lesion.

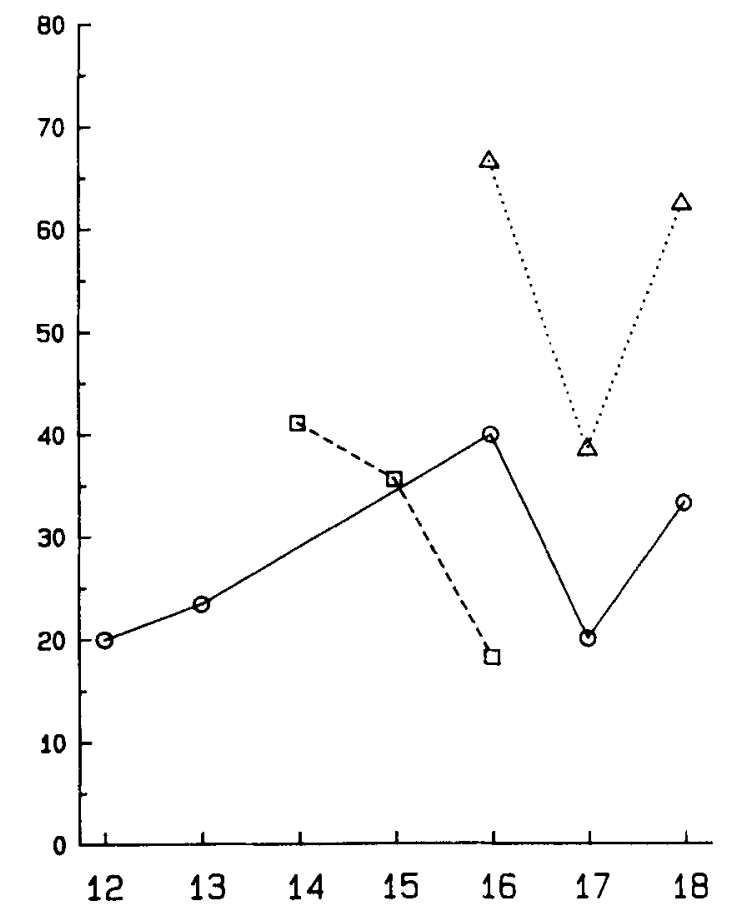

\section{BLOCKS Of 3 DAYS}

Fig. 6. Sequential testing of interrupt responses to coincident thermal stimuli delivered to cat $W$, first caudal (left) then rostral (right), to the thoracic spinal cord lesion. Reduced responsiveness to caudal stimuli is not attributable to effect of repeated testing. Test temperatures indicated in inset at left. The interrupt response profiles for this cat are shown in Figure 5. 
Response thresholds of cat $M$ were unchanged (interrupt) or increased (movement). Cats $\mathrm{L}$ and $\mathrm{S}$ both showed decreased interrupt thresholds postoperatively. There were no significant changes or differences in response latencies or laterality of any of the behaviors for any cat. No trends toward decreased or increased responsiveness could be detected by plotting response probabilities in sequential 3-day blocks for each cat. Furthermore, cat $\mathrm{M}$ showed reduced responses to hindlimb stimuli that were tested before testing rostral to the lesion.

All three cats with dorsal spinal lesions showed foodinduced modulation of interrupt responses to hindlimb stimuli. Figure 9 shows examples of the persistent response suppression in these cats. Cat $\mathrm{L}$ showed no modulation of movement responses during preoperative testing, so there is no information about the effect of the lesion on that response. However, cats $\mathrm{S}$ and $\mathrm{M}$ modulated both movement and interrupt responses to hindlimb stimuli.

\section{Effect of the lesions on tissue concentrations of neurotransmitter}

The concentrations of $5 \mathrm{HT}$ and $\mathrm{NE}$ in sections of the cervical and lumbar spinal cord were determined for seven normal unoperated cats and for each of the cats with partial lesions of the thoracic spinal cord. The results shown in Table 1 reveal a significant drop, compared to the unoperated control cats, in lumbar spinal cord 5HT concentrations only in the three cats with ventral lesions. There is also a highly significant decrease in Iumbar spinal cord NE in the cats with ventral lesions and a smaller, but statistically significant, decrease in lumbar NE in the two cats with the larger dorsal lesions.

Because these are matched samples of lumbar and cervical spinal cord from each cat, it is appropriate to consider the difference between the 5HT and NE concentrations at lumbar and cervical segments in each cat. The results, shown in Figure 10, reveal that both dorsal and ventral lesions produce significant lumbar-cervical differences in $\mathrm{NE}$ concentrations while significantly reduced lumbar $5 \mathrm{H} \mathrm{T}$ concentrations are seen only after ventral or the most extensive dorsal (cat S) lesions.

\section{DISCUSSION \\ Interpretation of the results}

We have presented evidence elsewhere (Casey and Morrow, '83) that the thermally elicited nocifensive behaviors we monitored are unlearned, nonreflexive responses that are evoked by activity in cutaneous $\mathrm{C}$ and, possibly, A $\delta$ afferent fibers ( $\mathrm{Li}$ et al., '85). We now offer the interpretation that the results of the experiments reported here are due to the effect of lesions of spinal sensory, rather than motor, systems mediating these behaviors. This is shown using Figure 11, which illustrates schematically the effect of thoracic spinal cord lesions on several types of supraspinal or suprasegmental ascending and descending systems.

In all cats, voluntary motor pathways (1, Fig. 11) to lumbosacral motor mechanisms were completely (cats $\mathrm{C}, \mathrm{W}$, and $\mathrm{P}$ ) or partially (cats S, L. and possibly M) destroyed. This interfered to varying degrees with the execution of voluntary lumbosacral movement (M, Fig. 11) but should have had little or no effect on voluntary movement or interrupt responses that are executed at cervical and lower brainstem levels (I, M, Fig. 11). Consequently, changes in the probability of these responses cannot be attributed to

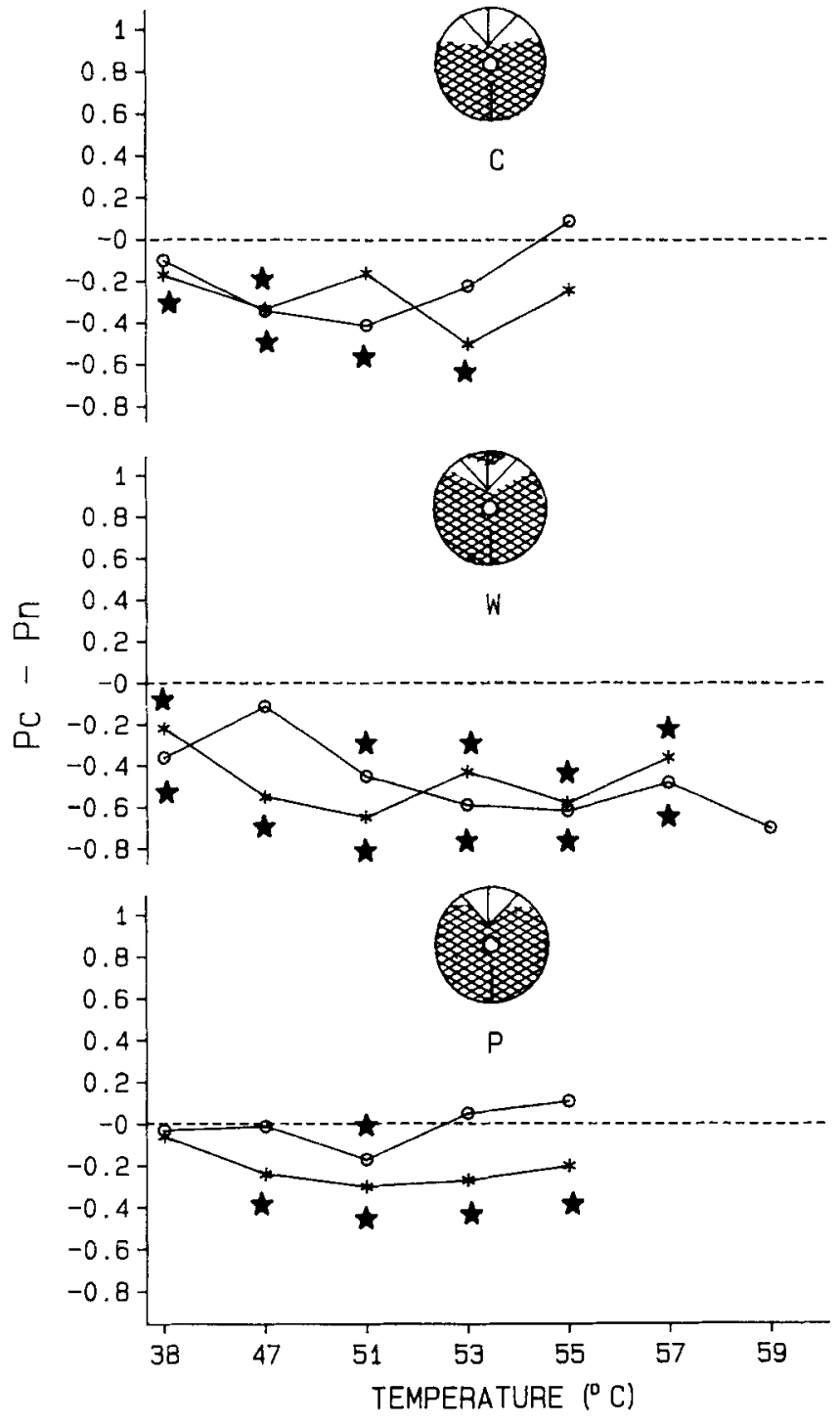

Fig. 7. Food-induced suppression of interrupt (cats $\mathrm{C}$ and $\mathrm{W}$ ) and movement (cat $P$ ) responses computed as in Figure 4. Asterisks show modulation of responses to stimuli delivered preoperatively to the hindlimbs (cats $\mathrm{C}$ and P) or rostral to the lesion (cat W). Open circles show modulation of responses to hindlimb stimuli caudal to the lesion (crosshatch in diagrams). Stars indicate that $(\mathrm{Pc}-\mathrm{Pn})$ exceeds the $95 \%$ confidence limits of the \% responses to both coincident and noncoincident stimuli.

TABLE 1. Concentration (ng/g tissue) of Serotonin and Norepinephrine in Cervical and Lumbar Segments of Whole Spinal Cord of Cats

\begin{tabular}{|c|c|c|c|c|}
\hline \multirow[b]{2}{*}{ Cat } & \multicolumn{2}{|c|}{ Serotonin } & \multicolumn{2}{|c|}{ Norepinephrine } \\
\hline & Cervical & Lumbar & Cervical & Lumbar \\
\hline $\begin{array}{l}\text { Control }^{1} \\
(\mathrm{~N}=7)\end{array}$ & $530 \pm 76$ & $764 \pm 106$ & $124 \pm 12$ & $183 \pm 17$ \\
\hline $\mathrm{C}$ & 363 & $144^{*}$ & 91 & $25^{* *}$ \\
\hline W & 532 & $49^{*}$ & 120 & $16^{* * *}$ \\
\hline $\mathrm{p}$ & 437 & $48^{*}$ & 105 & $8^{* *}$ \\
\hline $\mathrm{M}$ & 237 & 357 & 116 & 91 \\
\hline $\mathrm{S}$ & 516 & 375 & 83 & $73^{*}$ \\
\hline $\mathrm{L}$ & 345 & 310 & 73 & $63^{*}$ \\
\hline
\end{tabular}

${ }^{1}$ Control values expressed as mean $\pm \mathrm{SE}$ of seven cats.

$*=>-2 \mathrm{SD}$ from control mean concentration in lumbar segment.

$* *=>-3$ SD from control mean concentration in lumbar segment. 

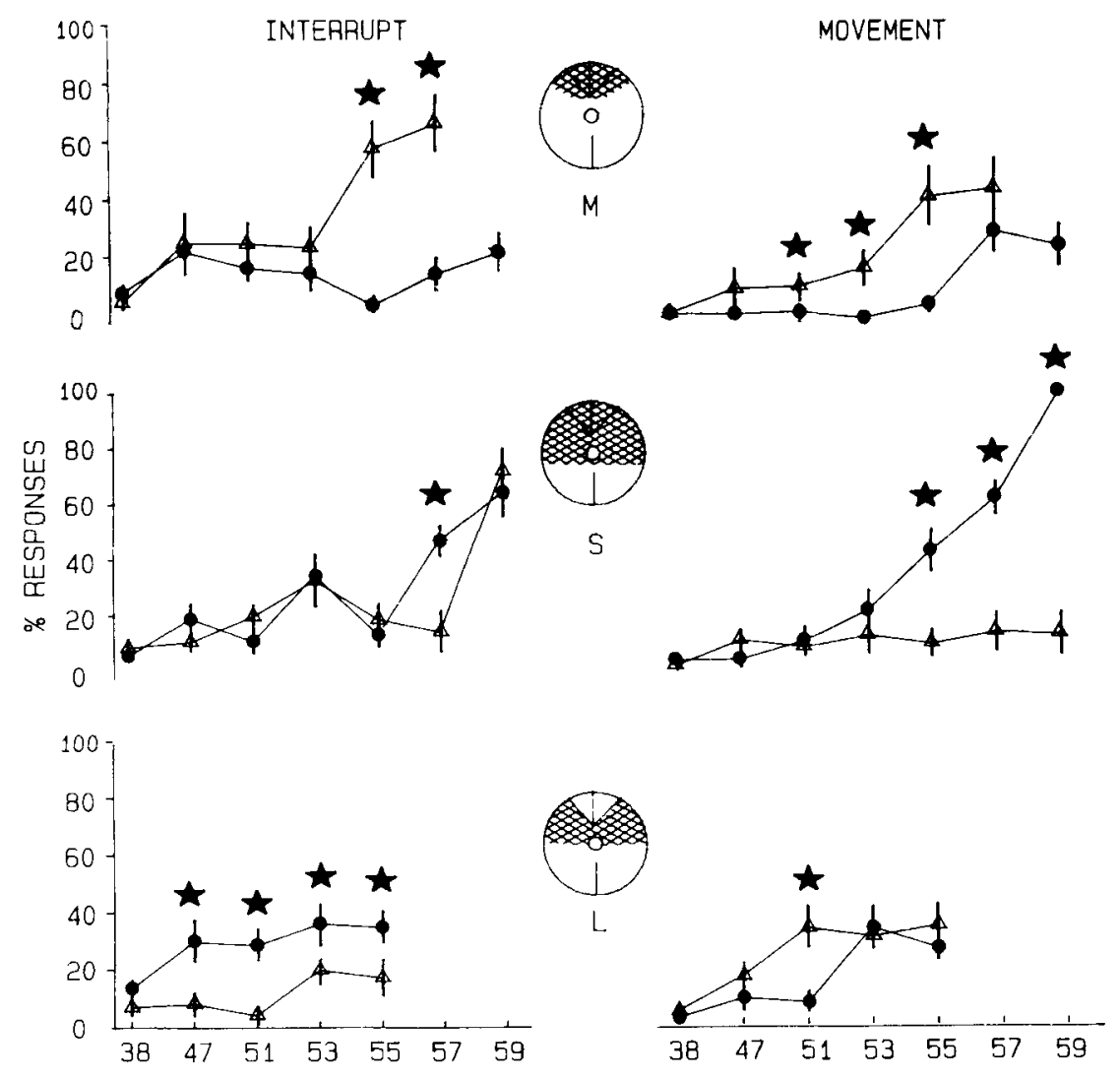

TEMPERATURE (OC)

\begin{abstract}
Fig. 8. Profiles of interrupt (left) and movement (right) responses to coincident thermal stimuli. Conventions same as in Figure 6. Cat $\mathbf{M}$ tested first caudal, then rostral to the lesion; cats $\mathbf{S}$ and $L$ tested on hindlimbs only. In this figure, all profiles show preserved responses to noxious thermal stimuli delivered caudal to the lesion. Note increased interrupt and movement responses of cat $S$ and interrupt responses of cat $L$.
\end{abstract}

motor deficits. This conclusion is supported by the lack of response latency changes after the lesions. The persistence of movement responses is attributable to the fact that they may be executed by cervical and supraspinal motor systems. We did not see thermally evoked movement responses due to segmental reflexes (dashed lines, Fig. 11) in the cat with a total transection of the thoracic spinal cord.

Interruption of ascending nociceptive sensory activity (3, Fig. 11) usually produces reduced nocifensive responses, as in most of these cats. It is also possible that lesions of ascending pathways could produce hyperresponsiveness by disinhibiting rostral motor responses. We cannot completely rule out the possibility that the increased movement responsiveness of cat $S$ was due to a partial lesion that disinhibited a segmental reflex (2, Fig. 11), but the interpretation that the increased movement responsiveness reflects changes in sensory activity is supported by the observation of concurrent increases in rostrally organized interrupt responses (cats $\mathrm{S}$ and $\mathrm{L}$ ) and the stability of response latency. Thus, hyperresponsiveness (cats $S$ and $L$ ) is probably due to the interruption of a tonic descending sensory inhibition ( $T$, 4, Fig. 11).

The tonic suppressive influence is clearly distinguishable from the phasic, food-induced modulation ( $P$, Fig. 11) because the phasic modulation survives lesions that remove the tonic inhibition. Indeed, the phasic modulation survives lesions that spare only the dorsal funiculi (cat P), suggesting that this form of modulation is organized primarily at supraspinal levels. The reduced modulation seen in cat $P$ may be due to the destruction of ascending or descending pathways.

Our results are attributable to the destruction or sparing of conducting pathways within spinal cord white matter rather than multisynaptic transmission within gray matter as reported by Karplus and Kreidl ('14) for the cat, Breazile and Kitchell ('68) for the pig, and Basbaum ('73) for the rat. Our large, bilateral lesions destroyed all or nearly all of the spinal cord gray matter at the lesion site in all but two cats ( $\mathrm{L}$ and $\mathrm{M}$ ), and the response changes observed in those two subjects must be attributable to white matter damage because all gray matter was spared.

Given the above arguments, we conclude that fibers ascending in either dorsal or ventral spinal funiculi are sufficient to elicit nonreflexive nocifensive behavior because lesions that spared fibers in either half of the spinal cord preserved such responses. Our results suggest that supraspinal nocifensive responses can be activated via the dorsal columns alone (cat P), but whether dorsolateral funicular pathways are sufficient cannot be determined from this study. 

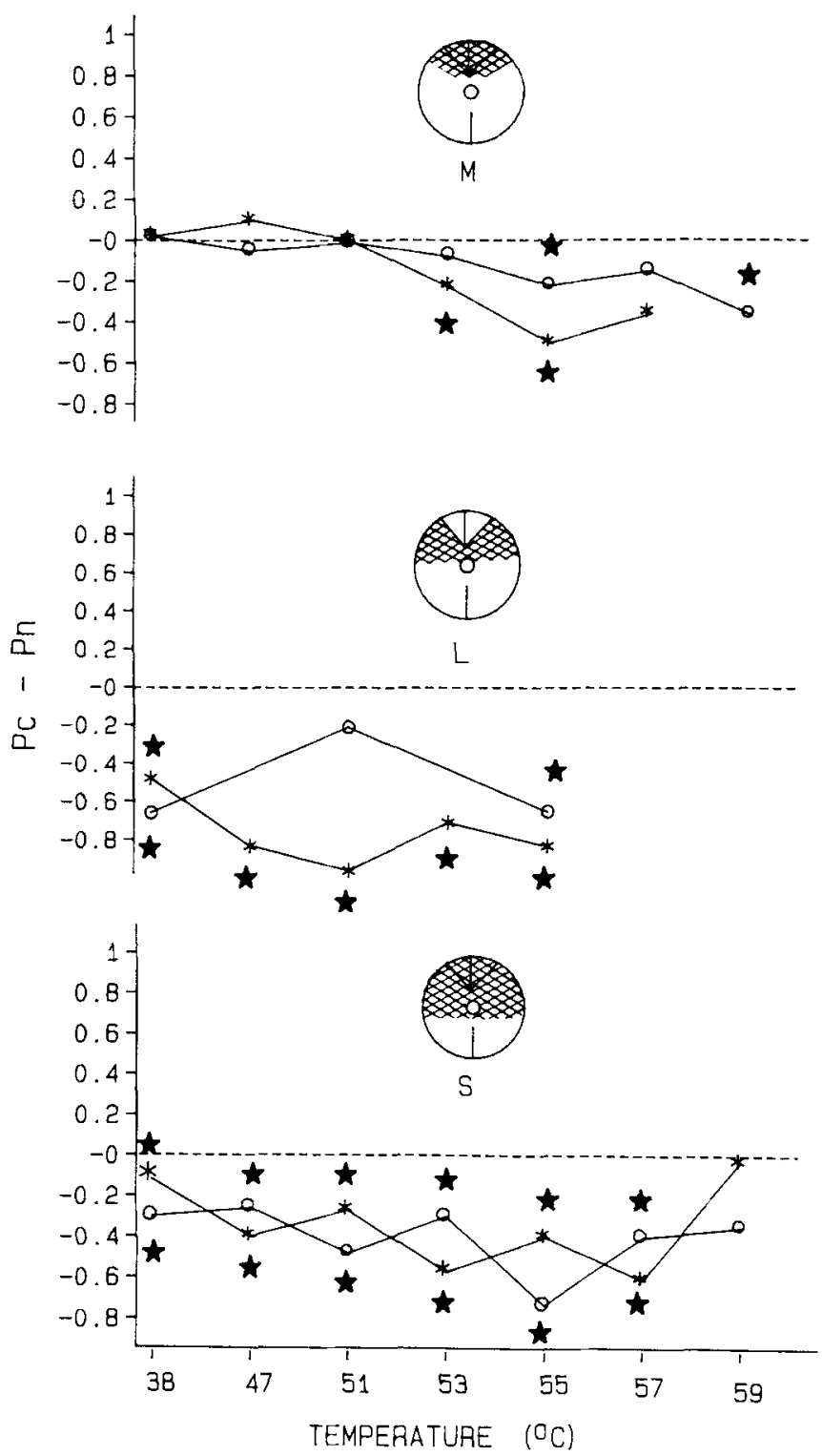

Fig. 9. Food-induced suppression of interrupt (cats $L$ and $S$ ) and movement (cat M) responses computed as in Figures 3 and 7. Conventions same as in Figure 7. Cat $M$ tested rostral and caudal to the lesion; cats $S$ and $L$ tested on hindlimbs only.

Both dorsal and ventral pathways are necessary to maintain normal responsiveness because response probability was significantly reduced after lesions involving either half of the spinal cord. The results of testing with tactile and noxious mechanical stimuli suggest that mechanical as well as thermal nocifensive mechanisms were affected by the lesions. However, in the mechanical stimulation tests, a loss of localizing and orienting capacity rather than a specific loss of nocifensive responsiveness cannot be ruled out (Levitt and Levitt, '68; Frommer et al., '77).

\section{Ascending pathways: Comparison with other studies in cat}

Kennard's conclusion that "the transmission of painful stimuli in the cat is chiefly via dorsolateral pathways" (Kennard, '54, p. 523) became especially salient because of the subsequent finding that neurons with axons ascending in the dorsal columns (Uddenberg, '68; Angaut-Petit, '75; Brown et al., '83; Lu et al., '83) or dorsolateral funiculus (Lundberg and Oscarsson, '61; Brown and Franz, '69; Brown et al., '75; Kniffki et al., '77) respond differentially or exclusively to noxious stimuli. Jones et al. ('85) and Hylden et al. ('86) have recently demonstrated, in the cat, dorsolateral spinothalamic and spinomesencephalic pathways originating primarily from dorsal horn neurons of lamina I, which is known to include a significant proportion of cells responding exclusively to noxious stimuli (Christensen and Perl, '70; Craig and Kniffki, '85). Our results strongly support the speculation that dorsolateral ascending pathways con tribute to the initiation of supraspinal nocifensive behaviors in the cat. The results obtained from cat $\mathrm{P}$ further suggest that fibers within the dorsal columns may be sufficient. Recent evidence that $29 \%$ of the primary afferent axons in the cat dorsal columns are unmyelinated (Chung and Coggeshall, '85) lends support to this speculation.

The function of dorsal nociceptive pathways remains to be determined. Our experiments required only the activation of a supraspinally organized nocifensive behavior, which could possibly be initiated by any pathway carrying nociceptive information. The prominent somatotopic organization of the dorsal funiculus and the observations of Levitt and Levitt ('68) suggest that dorsal nociceptive pathways might be important in orienting nocifensive responses towards the locus of stimulation (see also Dennis and Melzack, '77).

To the best of our knowledge, Kennard's ('54) is the only other behavioral study of the effect of ventral spinal cord tractotomies on nocifensive responses in the cat. She found no evidence for a pain-related sensory deficit in three cats with unilateral or two cats with bilateral ventral lesions. The extent of the lesions was not given, but in contrast with our results, none of Kennard's cats with ventral lesions had bladder disturbance; only one cat had a motor deficit, and that was unilateral. It is reasonable to assume, then, that our ventral lesions were more extensive than those produced by Kennard. Whether smaller ventral cordotomies would also attenuate nocifensive responses is not known. Nonetheless, there is agreement that ventral pathways are sufficient to maintain nocifensive responses because Kennard ('54) observed either a recovery or only a partial loss of pain-related responses in cats with bilateral dorsal lesions. These behavioral findings are also in accord with physiological studies of cats showing the transmission of nociceptive input via the ventral funiculi to the medullary (Casey, '69; Fields et al., '70, '75) and mesencephalic reticular formation (Yezierski and Schwartz, '86) and to the thalamus (Holloway et al., 78).

\section{Ascending pathways: Comparison with studies in other species}

Our results are consistent with studies indicating that, in other mammalian species, impulses ascending in both dorsal and ventral spinal cord pathways contribute to the 

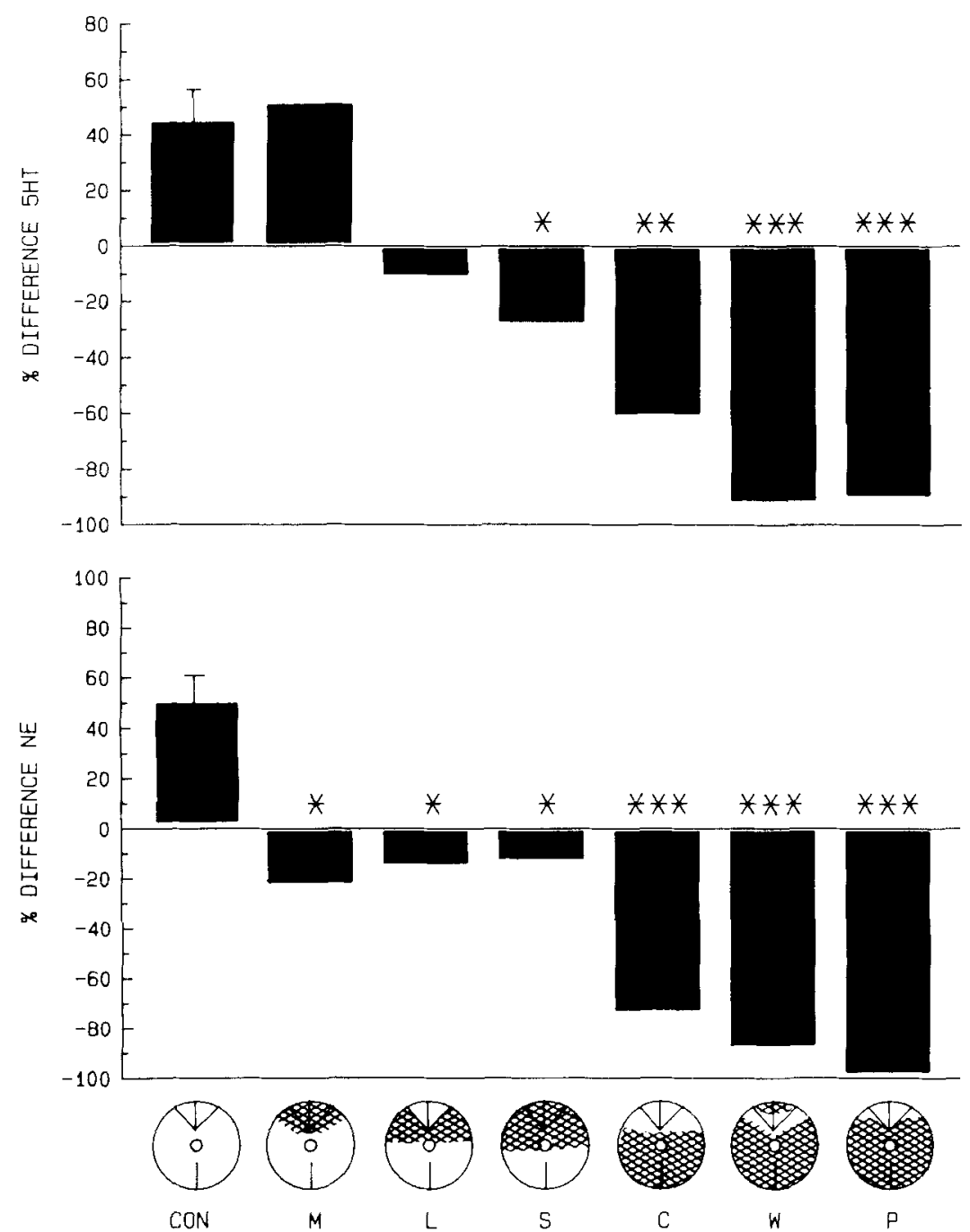

Fig. 10. Differences in 5HT (top) and NF (bottom) concentrations of matched paired samples of lumbar and cervical whole spinal cord. Measurements expressed as $\mathrm{ng} / \mathrm{g}$ tissue and converted to \% differences according to the formula: $\%$ difference $=$ (lumbar concentration - cervical concentration)/cervical concentration $\times 100$. Measurement from seven intact cats used as controls (CON, lower left diagram) show that the average $(+\mathrm{SE})$ lumbar

initiation of supraspinal nocifensive behavior in animals and to pain in humans. Breazile and Kitchell ('68) found that bilateral ventral lesions that spared only the dorsal and dorsolateral funiculi of the thoracic spinal cord preserved autonomic and electroencephalographic arousal responses to presumably noxious mechanical and thermal $\left(46-48^{\circ} \mathrm{C}\right)$ stimulation of the hindlimbs of the lightly anesthetized pig. These responses were eliminated by more extensive ventral lesions that spared only the dorsal columns. Vierck and Luck ('79) have shown that, in the monkey, bilateral ventral quadrant lesions of the thoracic spinal cord attenuate, but do not eliminate, upper limb operant escape responses to noxious electrical stimulation of the hindlimbs. Vierck et al. ('71) also demonstrated an attenuation of escape responses following dorsal column lesions in monkeys.
5HT and NE concentrations exceed that in the whole cervical spinal cord. Other differences refer to samples taken from cats identified by letter and lesion diagrams below each bar. Sample values lower than the average of the control sample by more than $2 \mathrm{SD}$ are marked by one asterisk; by more than $3 \mathrm{SD}$, by two asterisks; by more than $4 \mathrm{SD}$, by three asterisks.

In reviewing the clinical and anatomical data from 80 patients with ventrolateral spinal tractotomies, Nathan and Smith ('79) confirm that ventral ascending spinal cord pathways are necessary to maintain normal pain sensation in humans (see also Noordenbos and Wall, '76), but these authors also cite cases (Nathan, '56, '63) in which noxious stimuli, delivered caudal to a lesion that spares only the dorsal third of the spinal cord, elicit a burning pain referred rostrally to normally innervated areas. In an unusual case of amyotrophic lateral sclerosis in which only the dorsal columns were spared, pinprick sensation was reduced but preserved even though the anterolateral funiculi were almost completely destroyed (Scully et al., '79). Sweet (cited in Kerr and Casey, '78, pp. 149-150) has also presented clinical and anatomical evidence (Moosy et al., '67) that dorsal spinal cord pathways contribute to pain sensation in 
humans. In a recent extensive review of the function of dorsal spinal pathways in humans, Nathan et al. ('86) present evidence that dorsal column lesions may produce spontaneous pain, an increase or decrease in pain threshold, and an exaggeration of painful and thermal sensations. The authors also present evidence that lesions of the human dorsolateral funiculus alone produce no sensory deficits or abnormal responses to various innocuous stimuli, but tests for pain in these cases are not mentioned.

\section{Modulating influences}

Pavlov ('27) demonstrated, in classical conditioning experiments, that the presentation of food could eliminate the natural nocifensive responses of dogs to noxious stimuli. We have suggested (Interpretation of Results) that phasic, food-induced modulation may be primarily organized and executed at supraspinal levels because it survives extensive lesions that spare only the dorsal funiculi.

The neural mechanisms that tonically control responsiveness are clearly distinct from those subserving the phasic, food-induced modulation, which persists during hyperresponsiveness (cats $\mathrm{L}$ and $\mathrm{S}$ ). Because of the abundant evidence for a descending sensory control system in the dorsolateral funiculus (Basbaum, '78), it is reasonable to attribute the hyperresponsiveness to the interruption of such a path way in the cat (Soja and Sinclair, '83). It is known that anatomically and neurochemically distinct groups of neurons from the midbrain through the medulla can modulate the transmission of nociceptive and other somatososensory

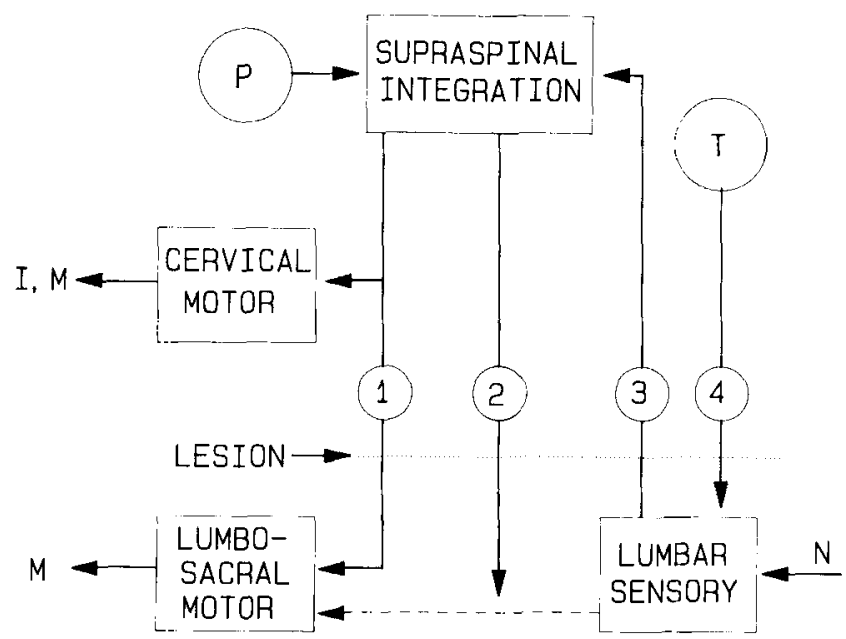

Fig. 11. Diagram of ascending and descending spinal systems that are pertinent to the interpretation of results. Thoracic spinal cord lesion may impair voluntary motor activation (1) by noxious stimuli (N) of movement responses $(M)$ executed at the lumbosacral level but should not affect inter rupt (I) or movement responses executed at cervical or lower brainstem levels. Destruction of an excitatory ascending nociceptive pathway (3), how ever, would reduce both types of nocifensive responses. Removing descend ing inhibition (2) of a segmental nocifensive reflex (dashed line) could produce increased lumbosacral movement responses, but this would not increase more rostrally organized interrupt behavior. Increased responsiveness following deep dorsolateral lesions is therefore probably due to removal of a tonic ( $T$ ) sensory inhibitory mechanism, which may be mediated by monoaminergic transmitters. Phasic (P) food-induced modulation is inde pendent of the tonic system and may be entirely supraspinal. See Discussion (Interpretation of Results) for more detailed explanation. information via axons descending primarily within the dorsolateral funiculus (for review see Mayer and Price, '76; Messing and Lytle, '77; Basbaum and Fields, '78; Basbaum, '82; Duggan, '82; Willis, '82; Vierck et al., '83). Most studies have used spinal segmental reflexive responses in rodents to assess the degree of presumed analgesia induced by direct electrical or chemical stimulation within the brain or induced by exposing the subject to various stressful stimuli. These reflexive measures of nociceptive activity are often unrelated to nonreflexive and operant nocifensive responses (Dennis et al., '80; Vierck et al., '83; Fasmer et al., '85; Jensen and Yaksh, '86). Nathan et al. ('86) did not state whether there were any changes in pain sensation or reactivity in humans with dorsolateral funicular lesions. Nonetheless, our results are consistent with the accumulated evidence for a descending nociceptive inhibitory system in the rodent dorsolateral funiculus and with the observations that lesions within the dorsolateral funiculus are associated with a significant reduction of the tail-flick latency in cats (Hayes et al., '84) and an increased reaction to noxious electrical stimuli in the monkey (Vierck et al., '71).

\section{Neurochemical changes: Relation to behavior}

The values of lumbar $5 \mathrm{HT}$ concentration we obtained before and after thoracic spinal cord lesions are in close agreement with similar measurements from whole spinal cord segments in the cat (Clineschmidt et al., '71). Our measurements of both cervical and lumbar $5 \mathrm{HT}$ and NE are also in accord with previous results obtained from rat, rabbit, and cat when differences among species and between whole cord and separate gray and white matter measurements are considered (Magnusson, '73; Zivin et al., '75; Oliveras et al., '77; Fleetwood-Walker and Coote, '81; Vasko et al., '84; Pang and Vasko, '86). The spinal cord concentration of NE, but not of $5 \mathrm{HT}$, reported for the rat by Janss et al. ('87) appears to be significantly higher than the value we obtained for the cat.

Our finding that lumbar spinal cord levels of both $5 \mathrm{HT}$ and NE in whole spinal cord sections are normally slightly higher than cervical levels is in agreement with the data obtained by others for the rat (Magnusson, '73; Zivin et al., '75; Vasko et al., '84). Similarly, the marked drop in the levels of both of those neurotransmitters caudal to large spinal cord lesions is to be expected (Clineschmidt et al., '71; Magnusson, '73). Significant reductions in 5 HT concentrations, however, were observed only after ventral lesions or large dorsal lesions that extended ventrally (cat S). The less extensive dorsal lesions produced a modest drop in lumbar NE concentrations but were not sufficient to affect lumbar 5HT levels significantly. These findings are consistent with the observation that monoamine-containing axons descend via both dorsal and ventral funiculi (Björklund and Skagerberg, '82; Stevens et al., '85). However, our re. sults may not reflect selective changes in neurotransmitter concentration in the dorsal or ventral gray matter.

Significant reductions of Iumbar spinal cord concentrations of both $5 \mathrm{HT}$ and NE were found in cats with preserved food-induced phasic modulation. The lack of relationship between spinal cord 5HT and NE levels and food-induced supression of responsiveness is not surprising if this phasic form of modulation is executed primarily or entirely at a supraspinal level, as our evidence suggests.

The dorsolateral lesions that produced hyperresponsiveness (cats $L$ and $S$ ) also resulted in a significant drop in 
lumbar NE concentrations compared to control samples (Table 1) and in a reversal of the normal control lumbar: cervical ratios of both $\mathrm{NE}$ and $5 \mathrm{HT}$ (Fig. 10). It is possible, then, that the interruption of the dorsal descending monoamine pathways, when combined with the sparing of most ascending ventral fibers, is sufficient to produce hyperresponsiveness to noxious stimuli. The selective sparing of dorsal and dorsolateral ascending pathways, however, may be sufficient only to maintain reduced nocifensive responses even though large ventral lesions have interrupted descending inhibitory $5 \mathrm{HT}$ and NE pathways. These hy potheses are in accord with the results of recent experiments (Mokha et al., '85; Aimone and Gebhart, '87; Janss et al., '87) and with current theories of monoamine-mediated descending analgesia mechanisms (Messing and Lytle, '77; Basbaum and Fields, '78; Yaksh and Hammond, '82; Dubner and Bennett, ' 83 ) but require additional experimental tests for verification.

\section{ACKNOWLEDGMENTS}

The authors thank Drs. L.C. Terry and R. Craig for performing the biochemical analyses and Dr. G.P. Frommer for cats and valuable advice. The careful technical assistance of Joyce Broka, Tena Casey, Larry Cronin, Pamela Graham, Bradley Hall, and Linda Sorkin is gratefully acknowledged. Patricia Morris assisted with graphics and data analysis.

Supported by grants from the National Institutes of Health (Public Health Service) and by the Veterans Administration.

\section{LITERATURE CITED}

Aimone, L.D., and G.F. Gebhart (1987) Spinal monoamine mediation of stimulation-produced antinociception from the lateral hypothalamus. Brain Res. 403:290-300.

Angaut-Petit, D. (1975) The dorsal column system: II. Functional properties and bulbar relay of the postsynaptic fibres of the cat's fasciculus gracilis. Exp. Brain Res. 22:471-493.

Basbaum, A.I. (1982) Anatomical substrates for the descending control of nociception. In B. Sjölund and A. Björklund (eds): Brain Stem Control of Spinal Mechanisms. Amsterdam: Elsevier, pp. 119-134.

Basbaum, A.1. (1973) Conduction of the effects of noxious stimulation by short-fiber multisynaptic systems of the spinal cord in the rat. Exp. Neurol. 40:699-716.

Basbaum, A.I. (1978) Three bulbospinal pathways from the rostral medulla of the cat: An autoradiographic study of pain modulating systems. J. Comp. Neurol. 178:209-224.

Basbaum, A.I., and H.L. Fields (1978) Endogenous pain control mechanisms: Review and hypothesis. Ann. Neurol 4:451-462.

Björklund, A., and G. Skagerberg (1982) Descending monoaminergic projec tions to the spinal cord. In B. Sjölund and A. Björklund (eds): Brain Stem Control of Spinal Mechanisms. Amsterdam; Elsevier, pp. 55-88.

Breazile, J.E., and R.L. Kitchell (1968) A study of fiber systems within the spinal cord of the domestic pig that subserve pain. J. Comp. Neurol. 133:373-382.

Brown, A.G., P.B. Brown, R.E.W. Fyffe, and L.M. Pubols (1983) Receptive field organization and response properties of spinal neurones with axons ascending the dorsal columns in the cat. J. Physiol. (Lond.) 337:575588 .

Brown, A.G., and D.N. Franz (1969) Responses of spinocervical tract neu rones to natural stimuli of identified cutaneous receptors. Exp. Brain Res. 7:231-249

Brown, A.G., W.D. Hamann, and H.F. Martin III (1975) Effects of activity in non-myelinated afferent fibres on the spinocervical tract. Brain Res. 98:243-259.

Casey, K.L. (1969) Somatic stimuli, spinal pathways, and size of cutaneous fibers influencing unit activity in the medial medullary reticular formation. Exp. Neurol. 25:35-56
Casey, K.L., B.R. Hall, and T.J. Morrow (1981) Effect of spinal cord lesions on responses of cats to thermal pulses. Pain, [Suppl.] 1:130 (abstract).

Casey, K.L., and T.J. Morrow (1983) Nocifensive responses to cutaneous thermal stimuli in the cat: Stimulus-response profiles, latencies, and afferent activity. J. Neurophysiol. 50:1497-1515.

Casey, K.L., T.J. Morrow, and L.C. Terry (1983) Pain suppression persists after interruption of descending serotonergic and noradrenergic pathways in cat spinal cord. Abstracts, 4th Annu. Meet. Am. Pain Soc., Chicago, Nov. 11-13, Am. Pain Soc., Nutley, NJ, p. 84.

Casey, K.L., T.J. Morrow, L.C. Terry, and R. Craig (1987) Differential effects of chronic partial myelotomies on monoamine levels in cat spinal cord. Brain Res. 408:377-380.

Chapman, C.R., K.L. Casey, R. Dubner, K.M. Foley, R.H. Gracely, and A.E. Reading (1985) Pain measurement: Ann overview: Pain 22:1-31.

Christensen, B.N., and E.R. Perl (1970) Spinal neurons specifically excited by noxious or thermal stimuli: Marginal zone of the dorsal horn. J. Neurophysiol. 33:293-306.

Chung, K., and R.E. Coggeshall (1985) Unmyelinated primary afferent fibers in dorsal funiculi of cat sacral spinal cord. J. Comp. Neurol. 238:365-369.

Clineschmidt, B.V., J.E. Pierce, and W. Lovenberg (1971) Tryptophan hydroxylase and serotonin in spinal cord and brain stem before and after spinal transection. J. Neurochem. 18:1593-1596.

Craig, A.D., and K.D. Kniffki (1985) Spinothalamic lumbosacral lamina I cells responsive to skin and muscle stimulation in the cat. J. Physiol. (Lond.) 365:197--222

Crow, E.L. (1956) Confidence intervals for a proportion. Biometrika 43:423435.

Dennis, S.G., and R. Melzack (1977) Pain-signalling systems in the dorsal and ventral spinal cord. Pain 4:97-132.

Dennis, S.G., R. Melzack, S. Gutman, and F. Boucher (1980) Pain modulation by adrenergic agents and morphine as measured by three pain tests. Life Sci. 26:1247-1259.

Dubner, R., and G.J. Bennett (1983) Spinal and trigeminal mechanisms of nociception. Annu. Rev. Neurosci. 6:381-418

Duggan, A.W. (1982) Brain stem inhibition of spinal transmission of nociceptive information: Pharmacologic studies of tonic and stimulationinduced inhibition. In B. Sjolund and A. Bjorklund (eds): Brain Stem Control of Spinal Mechanisms. Amsterdam: Elsevier, pp 439-450.

Fasmer, O.B., O.-G. Berge, and K. Hole (1985) Changes in nociception after lesions of descending serotonergic pathways induced with 5, 6-dihydroxytryptamine. Different effects in the formalin and tail-flick tests. Neuropharmacology 24:729-734.

Fellows, B.J. (1967) Chance stimulus sequences for discrimination tasks. Psychol. Bull, 67:87-92.

Fields, H.L., L.D. Partridge, Jr., and D.L. Winter (1970) Somatic and visceral receptive field properties of fibers in ventral quadrant white matter of the cat spinal cord. J. Neurophysiol. 33:827-837.

Fields, H.L., G.M. Wagner, and S.D. Anderson (1975) Some properties of spinal neurons projecting to the medial brain stem reticular formation. Exp. Neurol. 47:118-134.

Fleetwood-Walker, S.M., and J.H. Coote (1981) Contribution of noradrenaline-, dopamine-, and adrenaline-containing axons to the innervation of different regions of the spinal cord of the cat. Brain Res. 206:95-106.

Freund, J.E. (1962) Mathematical Statistics. Englewood Cliffs, NJ: Prentice Hall.

Frommer, G.P., B.R. Trefz, and K.L. Casey (1977) Somatosensory function and cortical unit activity in cats with only dorsal column fibers. Exp. Brain Res. 27:113-129.

Hayes, R.L., Y. Katayama, L.R. Watkins, and D.P. Becker (1984) Bilateral lesions of the dorsolateral funiculus of the cat spinal cord: Effects on basal nociceptive reflexes and nociceptive suppression produced by cholinergic activation of the pontine parabrachial region. Brain Res. 311:267-280.

Hekmatpanah, J. (1961) Organization of tactile dermatomes $\mathrm{C}_{1}$ through $\mathrm{L}_{4}$ in cat. J. Neurophysiol. 24:129-140.

Holloway, J.A., R.E. Fox, and A. Iggo (1978) Projections of the spinothalamic tract to the thalamic nuclei of the cat. Brain Res. 157:336-340.

Hylden, J.L.K., H. Hayashi, and G.J. Bennett (1986) Lamina 1 spinomesencephalic neurons in the cat ascend via the dorsolateral funiculi. Somatosens. Res. $41: 31-42$.

Janss, A.J., S.L. Jones, and G.F. Gebhart (1987) Effect of spinal norepinephrine depletion on descending inhibition of the tail flick reflex from the locus coeruleus and lateral reticular nucleus in the rat. Brain Res. 400:40-52. 
Jensen, T.S., and T.L. Yaksh (1986) Comparison of antinociceptive action of morphine in the periacqueductal gray, medial and paramedial medulla in rat. Brain Res. 363:99-113.

Jones, M.W., C.J. Hodge, Jr., A.V. Apkarian, and R.T. Stevens (1985) A dorsolateral spinothalamic pathway in cat. Brain Res. 335:188-193.

Karplus, J.P., and A. Kreidl (1914) Ein Beitrag zur Kenntnis der Schmer zleitung im Ruckenmark. Arch. Physiol. Pfluger 158:275-287.

Kennard, M.A. (1954) The course of ascending fibers in the spinal cord of the cat essential to the recognition of painful stimuli. J. Comp. Neurol. 100:511-524.

Kerr, F.W.L., and K.L. Casey (1978) Pain. Neurosci. Res. Prog. Bull. Vol. 16 Cambridge: MTT Press.

Kniffki, K.D., S. Mense, and R.F. Schmidt (1977) The spinothalamic tract as a possible pathway for muscular nociception. J. Physiol. (Paris) 73:359-366.

Levitt, M., and J. Levitt (1968) Sensory hindlimb representation in the Sml cortex of the cat after spinal tractotomies. Exp. Neurol. 22:276-302.

Li, C.Y., T.J. Morrow, and K.L. Casey (1985) Responses of single cutaneous fibers to noxious thermal pulse stimulation of cat upper hind limb. Pain $21: 17-24$.

Lu, G.W., G.J. Bennett, N. Nishikawa, M.J. Hoffert, and R. Dubner (1983) Extra- and intracellular recordings from dorsal column postsynaptic spinomedullary neurons in the cat. Exp. Neurol. 82:456-477.

Lundberg, A., and O. Oscarsson (1961) Three ascending pathways in the dorsal part of the lateral funiculus. Acta Physiol. Scand. 51:1-16.

Magnusson, T. (1973) Effect of chronic transection on dopamine, nonadrenaline and 5-hydroxytryptamine in the rat spinal cord. Naunyn Schmiedebergs Arch Pharmacol. 278:13-22.

Mayer, D.J., and D.D. Price (1976) Central nervous system mechanisms of analgesia. Pain 2:379-404.

Melzack, R., W.A. Stotler, and W.K. Livingston (1958) Effects of discrete brain lesions in cats on perception of noxious stimulation. J. Neurophysiol. 21:353-367.

Messing, R.B., and L.D. Lytle (1977) Serotonin-containing neurons: Their possible role in pain and analgesia. Pain 4:1-22.

Mokha, S.S., J.A. McMillan, and A. Iggo (1985) Descending control of spinal nociceptive transmission. Actions produced on spinal multireceptive neurones from the nuclei locus coeruleus (LC) and raphe magnus (NRM) Exp. Brain Res. 58:213-226.

Moosy, J., A. Sagone, and H.L. Rosomoff (1967) Percutaneous radiofre quency cervical cordotomy: Pathological anatomy. J. Neuropathol. Exp. Neurol. 26:118 (abstract).

Morrow, T.J., and K.L. Casey (1981) A contact thermal stimulator for neurobehavioral research on temperature sensation. Brain Res. Bull. 6:281284.

Nathan, P.W. (1956) Reference of sensation at the spinal level. J. Neurol. Neurosurg. Psychiatry 19:88-100.

Nathan, P.W. (1963) Resuits of antero-lateral cordotomy for pain in cancer. J. Neurol. Neursurg. Psychiatry 26:353-362

Nathan, P.W., and M.C. Smith (1979) Clinico-anatomical correlation in anterolateral cordotomy. In J.J. Bonica, J.C. Liebeskind, and D.G. AlbeFessard (eds): Advances in Pain Research and Therapy. New York: Raven Press, pp. 921-926.

Nathan, P.W., M.C. Smith, and A.W. Cook (1986) Sensory effects in man of lesions of the posterior columns and of some other afferent pathways. Brain 109:1003-1041.
Noordenbos, W., and P.D. Wall (1976) Diverse sensory functions with an almost totally divided spinal cord. A case of spinal cord transection with preservation of part of one anterolateral quadrant: Pain 2:185-196.

Oliveras, J.L., W. Bourgoin, F. Hery, J.M. Besson, and M. Hamon (1977) The topographical distribution of serotonin terminals in the spinal cord of the cat: Biochemical mapping by the combined use of microdissection and microassay procedures. Brain Res. 138:393-406.

Pang, I-G., and M.R. Vasko (1986) Effect of depletion of spinal cord norepinephrine on morphine induced antinociception. Brain Res. 371:171-176.

Pavlov, I.P. (1927) Conditioned reflexes. G.V. Andrep (editor and Translator). NY: Dover (1960), pp. 29-30.

Scully, R.E., J.J. Galdarini, and B.V. McNeely (eds) (1979) Case 46-1979. Case records of the Mass. Gen. Hosp. N. Engl. J. Med. 301:1104-1111.

Soja, P.J., and J.G. Sinclair (1983) Tonic descending influences of cat spinal cord dorsal horn neurons. Somatosens. Res. 1:83-93.

Stevens, R.T., A.V. Apkarian, and C.J. Hodge, Jr. (1985) Funicular course of catecholamine fibers innervating the lumbar spinal cord of the cat. Brain Res 336:243-251.

Terry, L.C., and R. Craig (1985) Cysteamine effects on monoamines, dopamine- $\beta$.hydroxylase and the hypothalamic-pituitary axis. Neuroendocrinology 41:46?-475.

Uddenberg, N. (1968) Functional organization of long, second order afferents in the dorsal funiculus. Exp. Brain Res. 4:377-382.

Vasko, M.R., I.-H. Pang, and M. Vogt (1984) Involvement of 5-hydroxytryptamine-containing neurons in antinociception produced by injection of morphine into nucleus raphe magnus or onto spinal cord. Brain Res. 306:341-348.

Vierck, C.J., Jr., B.Y. Cooper, O. Franzen, L.A. Ritz, and J.D. Greenspan (1983) Behavioral analysis of CNS pathways and transmitter systems involved in conduction and inhibition of pain sensations and reactions in primates. In J. Sprague and A. Epstein (eds); Prog. Psychobiol. Phys. iol. Psychol, Vol. 10. New York: Academic Press, pp. 113-165.

Vierck, C.J., Jr., D.M. Hamilton, and J.I. Thornby (1971) Pain reactivity of monkeys after lesions to the dorsal and lateral columns of the spinal cord. Exp. Brain Res. 13:140-158.

Vierck, C.d., Jr., and M.M. Luck (1979) Loss and recovery of reactivity to noxious stimuli in monkeys with primary spinothalamic cordotomies, followed by secondary and tertiary lesions of other cord sectors. Brain 102:233-248.

Willis, W.D. (1982) Mechanisms of medial brain stem stimulation-induced inhibition in spinothalamic neurons. In B. Sjöland and A. Björklund (eds): Brain Stem Control of Spinal Mechanisms. Amsterdam: Elsevier, pp. $411-438$.

Willis, W.D. (1985) The Pain System: The Neural Rasis of Naciceptive Transmission in the Mammalian Nervous System. Basel: Karger.

Willis, W.D., and R.E. Coggeshall (1978) Sensory Mechanisms of the Spinal Cord, NY: Plenum.

Yaksh, T.L., and D.L. Hammond (1982) Peripheral and central substrates involved in the rostrad transmission of nociceptive information. Pain 13:1-86.

Yezierski, R.P., and R.H. Schwartz (1986) Response and receptive field properties of spinomesencephalic tract cells in the cat. J. Neurophysiol. 55:76-96.

Zivin, J.A., J.L. Reid, J.M. Saavedra, and I.J. Kopin (1975) Quantitative localization of biogenic amines in the spinal cord. Brain Res. 99:293301. 